\title{
Imaging Motional Stark Effect measurements at ASDEX Upgrade
}

\author{
O. P. Ford, ${ }^{1}$ A. Burckhart, ${ }^{1}$ R. McDermott, ${ }^{1}$ R. Wolf, ${ }^{1}$ and the ASDEX Upgrade Team ${ }^{1}$
}

Max-Planck Institut für Plasmaphysik, Greifswald/Garching, Germany

\begin{abstract}
This paper presents an overview of results from the Imaging Motional Stark Effect (IMSE) diagnostic obtained during its first measurement campaign at ASDEX Upgrade since installation as a permanent diagnostic. A brief overview of the IMSE technique is given, followed by measurements of a standard H-mode discharge, which are compared to equilibrium reconstructions showing good agreement where expected. The development of special discharges for the calibration of pitch angle are reported and safety factor profile changes during sawteeth crashes are shown, which can be resolved to a few percent to due to the high sensitivity at good time resolution of the new IMSE system.
\end{abstract}

\section{Introduction}

Motional Stark Effect(MSE) polarimetry ${ }^{1}$ is one of the most productive diagnostic tools for current density profile determination in Tokamaks plasmas. MSE diagnostics measure the polarisation of $\mathrm{H} / \mathrm{D} \alpha$ emission from an injected beam of neutral hydrogen or deuterium. Typically, a Photo Elastic Modulator (PEM) and polariser temporally modulate the light intensity according to the polarisation state, which is measured by a photomultiplier tube or photodiode. A narrow interference filter selects either the $\sigma$ or $\pi$ component of the MSE multiplet to avoid cancellation due to their orthogonal polarisations. The requirement of separate detection hardware and filters for each channel, which must be individually tilt and temperature tuned to the Doppler shift, strongly limits the quantity of information that can be collected.

The Imaging MSE (IMSE) system $^{2}$ uses a $\mathrm{CCD} / \mathrm{CMOS}$ camera to capture a $2 \mathrm{D}$ image of the neutral beam and a set of birefringent plates and polariser to modulate the image spatially with interference patterns that encode the polarisation state. A typical raw image can be seen in figure 1a. The interference patterns have a strongly wavelength dependent phase, chosen such that all of the Stark multiplet transitions add constructively, despite their orthogonal polarisations. This removes the need for narrowband optical filters and allows the use of all of the available light. Coupled with the high speed, sensitivity and useful area of modern imaging cameras, the 2D image provides superior signal/noise and at least an order of magnitude more data than a typical MSE diagnostic.

A compact prototype IMSE system was developed ${ }^{3}$ for ASDEX Upgrade, thoroughly tested and installed in place of the classical MSE polarimeter for several weeks. After promising results ${ }^{4}$, the prototype system was installed on a new dedicated set of optics ${ }^{5}$. The calibration, first results and performance of the new system are documented here.

\section{Comparison to equilibrium predictions.}

Figure 1 shows a raw CCD image and demodulated polarisation angle image from one of the weekly standard H-mode discharges at ASDEX Upgrade. Since spatial resolution is in any case limited by integration across flux surfaces over the neutral beam width, a grid of $15 \times 12$ blocks is averaged and used for further analysis without significant loss of information. Figure 2 shows time traces of 6 blocks during a standard H-Mode shot. In total, the IMSE delivers at least 100 of these independent data points at the noise level shown, an order of magnitude improvement over the traditional MSE systems.
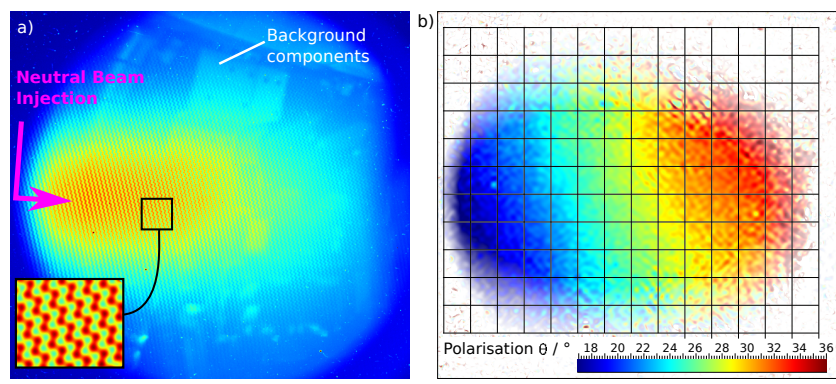

FIG. 1. a) Raw IMSE image and b) demodulated polarisation angle image for a single time point of a reference $\mathrm{H}$-mode discharge. The polarisation angle image is intensity blended according to the uncertainty estimate calculated from CCD shot-noise.

As no in-vessel absolute calibration could be performed due to time constraints, the time traces are arbitrarily offset to highlight the good agreement of the time evolution with that predicted from an equilibrium reconstruction (magnetics-only). Significant deviation is seen only at the end of the discharge when sawteeth crashes appear, which the equilbrium code is unable to predict. The predictions include the effects of Faraday rotation and of the radial electric field.

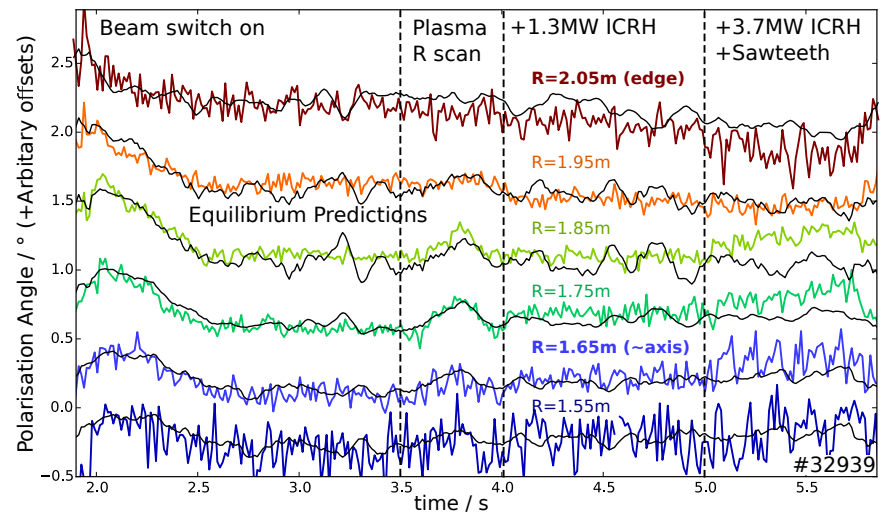

FIG. 2. Time evolution of measured polarisation angle at 6 locations during a reference H-Mode discharge. Also shown are predictions from the equilibrium code CLISTE (magnetics only) including Faraday rotation and $E_{r}$ corrections. Offsets of traces are set arbitarily to match. 
Figure 3 shows a profile taken from the image (blue) versus major radius compared with the equilibrium prediction. In this case, adjusting the prediction by a linear function of radius is sufficient and the remaining curvature matches that calculated from the equilibrium. The profile of the difference between two time points (shown in black), which show the change due to switch-on of the neutral beam, requires no offset and shows good agreement with the prediction.

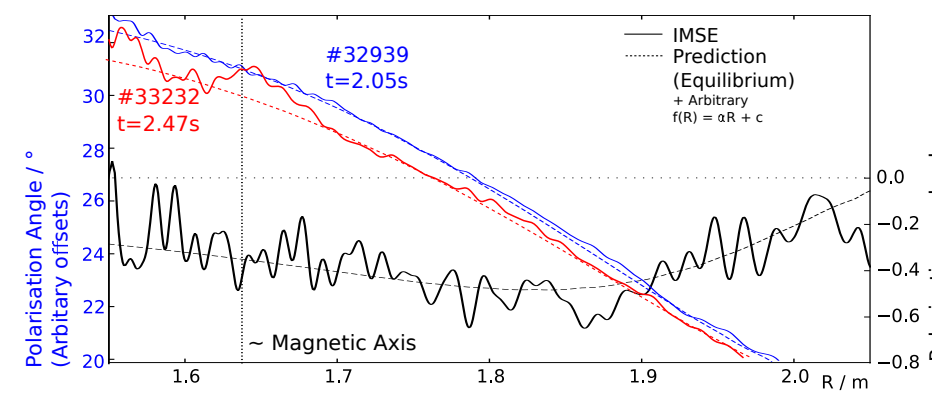

FIG. 3. Blue: Measured polarisation profile in a good reference H-Mode discharge, with equilibrium prediction (modified by an arbitrary linear offset). Black: Prediction and measurement of difference between $t=3.00 \mathrm{~s}$ and $\mathrm{t}=2.05 \mathrm{~s}$. Red: Later discharge showing disturbances at the core.

For much of the first campaign, the core data $(R<$ $1.7 \mathrm{~m})$ is disturbed, most likely by a reflection in the optics. A critical possibility, the complex interaction of the spectral optimisation as described in $^{3}$, was excluded by showing that the spectral distribution, which was modified by changes of beam energy and band-pass filter incidence angle, does not alter it. As the disturbance may therefore involve contamination with light from a different position, data in $R<1.7 \mathrm{~m}$ cannot be trusted until this is solved. Other than this, the very good agreement of the dynamics indicates that at least for small changes, no sigificant systematic problems are present.

\section{Reverse Bt calibration}

The measured polarisation angle $\theta$ is approximately linearly related to the pitch angle by the pitch angle sensitivity $\alpha$, which is determined from the beam and view geometry. For the permanent IMSE system ${ }^{5}$ this lies in the range $0.4<\alpha<0.6$.

$$
\theta-\theta_{c} \approx \alpha B_{z} / B_{\phi}
$$

In order to calibrate the offset $\theta_{c}$, a pair of otherwise similar discharges were developed with normal and reversed toroidal field $B_{\phi}$ but the same plasma current direction. The average of the two shots should approximately give $\theta_{c}$, the polarisation of zero pitch and the position at which the polarisation is unchanged by reversal of $B_{\phi}$ should be that of the magnetic axis. To check this, the magnetic axis position is scanned slowly over the range $1.70<R_{0}<1.74 m$. Figure 4 shows the polarisation profiles recorded during the position scan for both positive and negative $B_{\phi}$.

Again, the effect of the disturbance can clearly be seen for $R<1.7 m$, particularly for the normal $B_{\phi}$ direction. Outside $R \approx 1.87 \mathrm{~m}$, the clear linear movement of

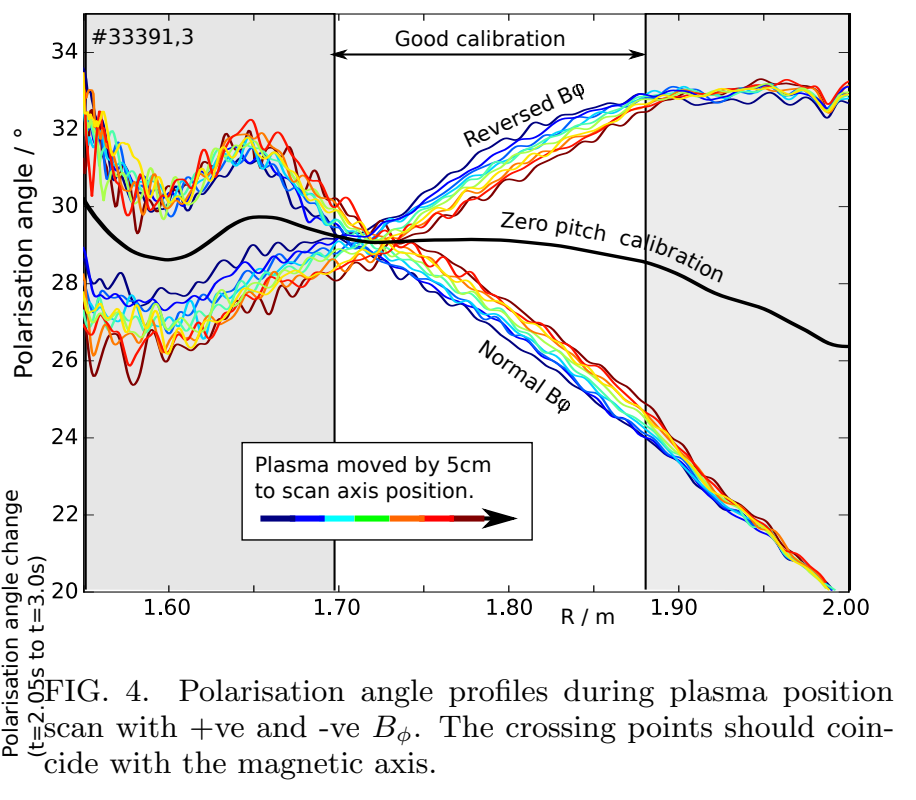

the plasma is no longer seen and the calibration method should not be considered valid. At this distance from the axis, the linear dependence on the pitch angle is no longer valid. Additionally, the linearity of the diagnostic can not be trusted over the $10^{\circ}$ difference due to the as-yet uncompensated intrinsic contrast effect $\left(\mathrm{see}^{3}\right)$. Within the core region, the radial position of the crossing point can be seen to move outward during the position scan. Figure $5 \mathrm{a}$ shows the surprisingly good agreement between the crossing point as determined from a linear fit of each profile within $1.69<R_{0}<1.78 m$, with that predicted by the equilibrium code.
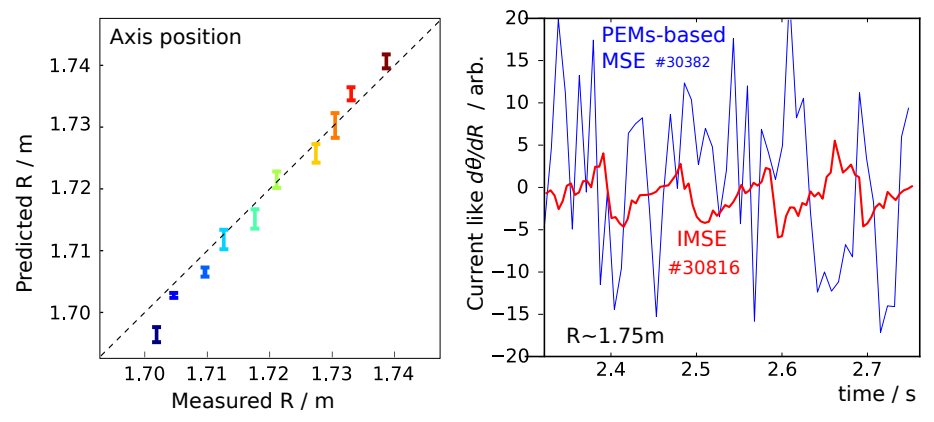

FIG. 5. a) Magnetic axis position from equilibrium vs crossing point of measured profiles for normal and reversed toroidal field. Prediction is the range between normal and reversed $B_{\phi}$ discharges. b) Current-like quantity from the difference between polarisation angle of two radial channels of the PEM based MSE polarimeter (blue) and of equivalent areas of the IMSE image (red) near the plasma core during sawteeth.

As the field line pitch angle of the reversed $B_{\phi}$ is incompatible with the lower divertor, reversed $B_{\phi}$ discharges at ASDEX Upgrade must use an upper single null plasma. In this configuration, the axis position scan can not cover the usual axis position of $R \approx 1.65 \mathrm{~m}$, where the calibration is most desirable, but the disturbance currently prohibits calibration in this region in any case. The upper single null configuration also uses the rarely used open upper divertor, making it difficult to control the plasma 
density and impurity content and hence to produce identical shots. Finally, the L-H transistion threshold in the upper-single null configuration is higher for normal $B_{\phi}$ than reversed due to the unfavourable grad-B drift direction. To achieve similar conditions in both discharges, care must be taken that both remain in H-mode and that the initial transisition is made rapidly.

\section{Sawtooth Dynamics}

The large quantity of low noise measurements allows the inference of very small changes in the current profile, such as during the sawtooth $\mathrm{crash}^{6-8}$. With a reliable calibration proceedure, it will be possible to include the IMSE data as constraints in an equilibrium reconstruction. However, it is difficult to see in such reconstructions what is inferred from the data, and what comes from the assumptions of the equilbrium code. Instead, an approximation of the toroidal current density $j_{\phi}$ can be calculated directly ${ }^{9}$ from the radial derivative of $B_{z}$ (equation 2). $B_{z}$ is determined from the polarisation angle $\theta$, the calibration $\theta_{c}$ (figure 4 , black) and the vacuum toroidal field $B_{\phi}$ using equation 1 . The elongation profile $\kappa(R)$ can be taken from the standard equilibrium reconstruction as $\kappa$ is a weak function of $R$ and does not change significantly in time.

$$
\mu_{0} J_{\phi}=-\left(1+\frac{1}{\kappa^{2}}\right) \frac{\partial B_{z}}{\partial R}
$$

The quantity of most interest in the sawtooth crash is the central safety factor $q_{0}{ }^{6-8}$. From the large aspect ratio approximation $q_{0} \sim 2 B_{\phi} / \mu_{0} j_{\phi} R_{0}$, it can be seen that small changes $\Delta q_{0}<<1$ near $q_{0} \sim 1.0$ (as are expected for sawteeth) are approximately linearly related to the radial derivative of the polarisation angle. To illustrate the importance of the IMSE's high sensitivity, figure 5b compares the difference between neighbouring core channels $(\Delta R 4 \mathrm{~cm})$ of the MSE polarimeter to the difference between averages of equivalent areas of the IMSE image. While changes can be clearly seen in the IMSE signal, the signal/noise of the PEM based MSE is far too low to resolve them. IMSE data from a shot with large consistent sawteeth were averaged into 10 profiles according to their phase in the sawtooth cycle as determined from the soft X-ray diagnostic. With correction for Faraday rotation and radial electric field, current density profiles derived using equations 1 and 2 are shown in figure 6 . The redistribution of current at the crash from the core region to outside an inversion radius of $R \sim 1.83 \mathrm{~m}$ is very clear. This is followed by a gradual rebuilding of the central current density. The absolute value and profile shape of the core region cannot yet be used due to the disturbance.

If the current density profile is not strongly peaked at the magnetic axis, the large aspect approximation for $q_{0}$ remains approximately valid in the vicinity, at least well enough to show the magnitude of changes in $q$. Figure 7 shows $\Delta q$ at the location of strongest change $R=1.77 \mathrm{~m}$ to demonstrate that at a spatial resolution of $5 \mathrm{~cm}$ and time resolution of $5.7 \mathrm{~ms}$, the IMSE has sufficiently low noise to resolve dynamics of the safety factor profile $\Delta q$ to within a few percent, in profiles derived directly from
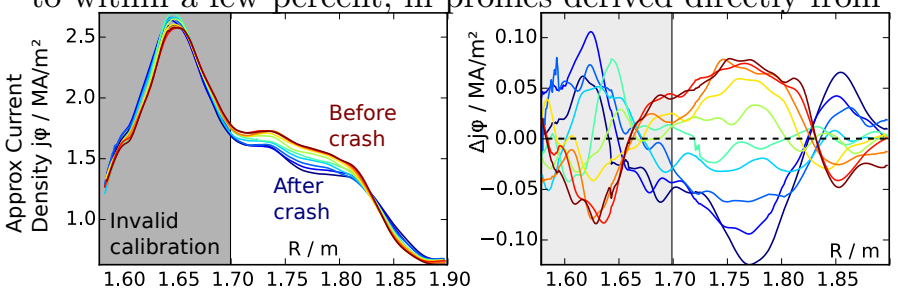

FIG. 6. a) Evolution of the toroidal current density profile $j_{\phi}$ during sawteeth as derived from IMSE polarisation angle images using equation 2. Each profile is the average of $1 / 10$ th of the sawtooth cycle over 40 sawteeth crashes. Spatial smoothing with length scale $4 \mathrm{~cm}$ is also applied to reduce noise. b) Profiles relative to the average, showing the maximum change.

the raw data. This represents a significant improvement in the diagnostic capability of core Tokamak current density. In future work, the forward/reverse $B_{\phi}$ calibration method will be further developed and compared with an absolute in-vessel calibration. A new back-end will also be installed to improve the diagnostic stability and sentivity even further.

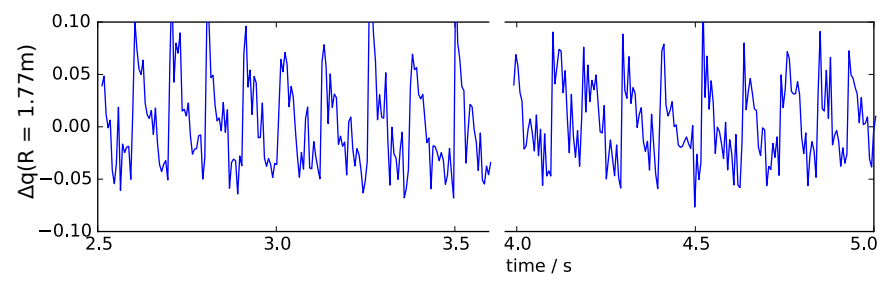

FIG. 7. Approximate changes in $q$ at $R=1.76 \mathrm{~m}$. The values are only indicative of the magnitude of changes due to the strong assumptions made in the calculation.

This work has been carried out within the framework of the EUROfusion Consortium and has received funding from the Euratom research and training programme 2014-2018 under grant agreement No 633053. The views and opinions expressed herein do not necessarily reflect those of the European Commission.

${ }^{1}$ F.M. Levinton, R.J. Fonck, G.M. Gammel, R. Kaita, H.W. Kugel, E.T. Powell, and D.W. Roberts, Phys. Rev. Lett. 63, 2060 (1989).

2 J. Howard, Plasma Physics and Controlled Fusion 50, 125003 (2008).

${ }^{3}$ O.P. Ford, J.Howard and R.C.Wolf,Rev. Sci. Instrum. 86, 093504 (2015).

${ }^{4}$ O.P. Ford et al., in 40th Europhysics Conference Proceedings, Espoo, Finland, O2.110 (2013).

${ }^{5}$ A. Burckhart et al., in Europhysics Conference Proceedings, Lisbon, Portugal., P1.143 (2015).

${ }^{6}$ D. Wróblewski and R.T. Snider, Phys. Rev. Lett. 71, 859 (1993).

${ }^{7}$ M. Yamada et al., Rev. Sci. Instrum. 63 (1992).

${ }^{8}$ H. R. Koslowski, Fusion science and technology 47, 260 (2005).

${ }^{9}$ C. Petty et al., Nuclear Fusion 42, 1124 (2002). 